\title{
DIQUARK STRUCTURE IN HEAVY QUARK BARYONS IN A GEOMETRIC MODEL
}

\author{
Lina Paria and Afsar Abbas \\ Institute Of Physics \\ Bhubaneswar-751005 \\ India \\ e-mail : lina@iopb.ernet.in \\ e-mail: afsar@iopb.ernet.in
}

\begin{abstract}
Using a geometric model for the study of the structure of hadrons, we study baryons having one, two and three heavy quarks. The study reveals diquark structure in baryons with one and two heavy quarks but not with three heavy identical quarks.
\end{abstract}


The study of the heavy quark systems $[1]$ is an important issue in particle physics. It has even become more so in the recent years [2] as several existing and planned machines are expected to produce a barrage of experimental information in the near future. The field has received a boost from the so called Heavy Quark Effective Theory [3, 4, 5]. An interesting property which arises in this theory is the existence of the diquark structure for two heavy quarks (QQ) in a baryon consisting of $Q Q q$, (where $q$ is for a light quark) [6, [].

Now the study of diquark is almost as old as that of the quarks. Existance or nonexistance of diquark structure in baryons with (i) $q q q$, (ii) $q q Q$, (iii) $q Q Q$, (iv) $Q Q Q$ has been a problem of much interest [8, 9] and has recently been reviewed [10]. Most studies have been done within the framework of potential models, bag models and string models [10. The cases (i), (ii) and (iii) have been well studied but not the case (iv). In our study in addition to the study of the cases (i), (ii), (iii) we shall do case (iv) as well. However the information extracted as to the diquark structures obtained by different people are often in direct conflict with each other. Some consensus has been achieved but there is much confusion. The situation can be best summerised by quoting the statement of the "five" authors of the review article [10" "....sometimes we do not agree among ourselves about the nature of diquarks".

Our aim in this paper is to study this important dual problem of the heavy quarks and of the diquark structure in baryon within a framework which is complementary to the potential models and the bag models pictures. This is a geometric model made use of recently [11, 12] in the context of light baryons. 
In fact a diquark structure was obtained therein [12]. This view complements and supports the view that the nucleon is deformed in the ground state. This was obtained within the configuration mixed wave function picture in a quark potential model [13].

In the geometric model of hadrons quarks sit at different positions in a collective picture [11, 12]. All the excited states of hadrons are obtained by rotations and vibrations of quarks in a collective mode. For simplicity, we will be considering only the rotational excitations. The total wave function of baryons is given by

$$
\psi_{\text {total }}=\psi_{c} \otimes \psi_{s f} \otimes \psi_{r}
$$

where $\psi_{c}, \psi_{s f}$ and $\psi_{r}$ stands for the wave function corresponding to color, spin-flavor and the geometric degrees of freedom respectively. From the antisymetrisation of the color state, the product of the wave function $\psi_{s f} \otimes \psi_{r}$ has to be totally symmetric.

To understand the geometric structure of baryons, we are considering the three possible geometrical arrangements of quarks as in Fig. 1. Here general case (a) : two quarks are seperated by a distance $r_{2}$, while the third quark is at a distance $r_{1}$ from the center of mass of the first two quarks. case (b) $: r_{2}=0$; i.e. two quarks are at the same place while the third one is separated. case (c) $: \frac{r_{1}}{r_{2}}=\frac{\sqrt{3}}{2}$; i.e. three quarks are sitting at the vertices of an equilateral triangle. case (d) $: r_{1}=0$; i.e. all quarks are equidistant and lying on a line. The point group structure for inertia tensor $\left(G_{I}\right)$, for equivalent particles $\left(G_{E}\right)$ and for distinct particles $\left(G_{D}\right)$ are given below 
respectively.

$$
\begin{aligned}
\text { general case }(a) & : C_{2 v}, C_{2 v}, S_{1} \\
\operatorname{case}(b) & : C_{\infty v}, C_{\infty v}, C_{\infty v} \\
\operatorname{case}(c) & : D_{3 h}, D_{3 h}, S_{1} \\
\operatorname{case}(d) & : D_{\infty h}, D_{\infty h}, C_{\infty v}
\end{aligned}
$$

As we are considering baryons with a heavy flavour ( charm) the corresponding spin flavor group symmetry is $S U_{s f}(8)$. The group $S U_{s f}(8)$ is broken in the following chain of subalgebras

$$
\begin{aligned}
S U_{s f}(8) & \supset S U_{f}(4) \otimes S U_{s}(2) \\
& \supset S U_{f}(3) \otimes U_{c}(1) \otimes S U_{s}(2) \\
& \supset S U_{I}(2) \otimes U_{Y}(1) \otimes U_{c}(1) \otimes S U_{s}(2) \\
& \supset S O_{I}(2) \otimes U_{Y}(1) \otimes U_{c}(1) \otimes S O_{s}(2)
\end{aligned}
$$

The decomposition of the relevant representations of $S U_{s f}(8)$ into $S U_{f}(4) \otimes S U_{s}(2)$ are:

$$
\begin{array}{rll}
120 & \supset & { }^{4} 20 \oplus^{2} 20 \\
168 & \supset & { }^{2} 20 \oplus{ }^{4} 20 \oplus^{2} 20 \oplus^{2} \overline{4} \\
56 & \supset & { }^{2} 20 \oplus{ }^{4} \overline{4}
\end{array}
$$

The quantum numbers of the baryons $N, \Delta, \Lambda_{c}, \Sigma_{c}, \Xi_{c c}, \Omega_{c c c}$ under $S U_{s f}(8)$ group symmetry are given in Table I. Fig. 2 is the schematic diagram drawn on the basis of table 2 in ref [12]. This shows the variation of levels with a change in the shape of the baryons (as stated in the cases $((b),(c),(d))$ ) 
for $L=0 \& L=1$. Here $L$ is the orbital angular momentum and $K$ is the projection of the orbital angular momentum on the body fixed axis with $L=K, K+1, K+2, \ldots \ldots$ The notation used here is : $K=0+$ indicate $L=0,2,4, \ldots$ and $K=0$ - indicate $L=1,3,5, \ldots \ldots$ This diagram is a slightly modified version of the one by Halse [12].

Note that the spectrum generating algebra chain in equation (3) implies that the symmetry is broken diagonally. ( It is because of this, that the fact that the c- quark is much heavier than the $\mathrm{u}^{-}, \mathrm{d}_{-}$, and $\mathrm{s}^{-}$quarks is not expected to effect our analysis ). This means that the energy levels may depend on the eigen value of the Casimir operators of the group chain. Hence the $S U(8)$ mass formula is

$$
\begin{aligned}
M^{2} & =M_{0}^{2}+a\left[\hat{C}_{2}(S U(8))-\frac{231}{8}\right] \\
& +b\left[\hat{C}_{2}(S U(4))-\frac{39}{4}\right]+c\left[\hat{C}_{2}(S U(3))-\frac{8}{3}\right]+d\left[s(s+1)-\frac{3}{4}\right] \\
& +e[C-1]+f[I(I+1)]+\alpha L+\beta K
\end{aligned}
$$

where $\hat{C}_{2}$ is the eigenvalue of the Casimir operator for different representation, $C$ is charm quantum number, $s$ is spin value, $I$ is isospin, and $a, b, c$, $d, e, f, \alpha, \beta$ are parameters. The term $\alpha L$ and $\beta K$ in the mass formula comes from the rotation of the system. The operators are defined in such a way that for the ground state of $\Lambda_{c}$ all the terms except $M_{0}^{2}$ vanish. The justification for using the linear term in $L$ in the above formula arises from the fact that our model is intrinsically related to the stringlike bag model (as evident from Fig. 1 ). This has also been pointed out in ref.[11].

We treat Kalman and Tran's result [14] on the heavy quark baryons as a good representative sample of the theoretical studies in this area. In addition 
these results are published in a great detail making them suitable as a point of reference. We will treat these numbers as "experimental numbers". Note that we do not consider $\Xi_{c}, \Omega_{c}, \Omega_{c c}$ etc. as the corresponding data does not exist. We do not expect much changes in our basic conclusions when the real experimental numbers become available. The available experimental data is from [15].

For each baryons, we arrange the states in such a way that the energy will increase from lower excited states to higher excited states. Knowing the internal quantum numbers, spin-flavour symmetry of each baryon considered is given in Table I . Knowing the Casimir operator value of the relevant representations and using the mass formula we can specify each states of these baryons in a particular $[g] L^{\pi}$ representation. Here $\pi$ is the parity of the state and $[g]$ is the $S U(8)$ representation in the standard Young Diagram formalism giving the total number of boxes in each row, e.g. [21] denote the two boxes in first row and one box in second row. The notation $\left(p_{1} p_{2} p_{3}\right)$ in Table I denote the difference in the number of boxes between the rows. i.e $p_{i}=\lambda_{i}-\lambda_{i+1}$, where $\lambda_{i}$ is the number of boxes in the $i^{t h}$ row of the Y.D. Our approach for assignment of the states of baryons is that of Halse [12] where the mass formula is used as a guide for these assignments.

As an example, for $\Lambda_{c}$ :

(i) The lowest energy state $J^{\pi}(M)$ is $\frac{1}{2}^{+}(2282)$, with $L=0, s=\frac{1}{2}, K=0$, ( 1110 ) representation of $S U(4)$, ( 01 ) representation of $S U(3)$. Now using the Casimir operator value of each representation in the mass formula we get $M^{2}=M_{0}^{2}$ and the state $\frac{1}{2}^{+}(2282)$ goes to the $[g] L^{\pi}$ representation as $[3] 0^{+}$. 
(ii) The next excited state $J^{\pi}(M)$ is $\frac{1}{2}^{-}(2653)$, with $L=1, s=\frac{1}{2}, K=0$, ( 1110 ) representation of $S U(4)$, ( 01 ) representation of $S U(3)$. This assigns this state in the $[g] L^{\pi}$ representation as $[3] 1^{-}$( which gives a reasonable fit ) rather than the assignment $[21] 1^{-}$and so on. In this way the states of each baryons considered in Table I are assigned and a global fit of the states are able to give the parameter values as : $M_{0}^{2}=6.303, a=-0.317, b=0.163$, $d=-0.152, \alpha=1.297, \beta=1.022, c=9.5025, e=18.7795, f=-18.994$. All parameters are in unit $G e V^{2}$.

The low excited states of $N, \Delta, \Lambda_{c}, \Sigma_{c}, \Xi_{c c}, \Omega_{c c c}$ have been given the assignment shown in Table II. The location of these particles as per our assignment is also indicated in Fig. 2.

We find that the nucleon has the structure falling in between the case(b) and (c) ( Fig. 2.). The position of $\Delta$ is found to be slightly to the right of $N$ (see Fig. 2.). This is in contrast to what Halse [12] had obtained for $N$ and $\Delta$. This is because in his fit he was trying to include a single star state $\frac{1}{2}^{+}(1550)$ which existed then (i.e. in the 1986 data set), but does not exist anymore ( i.e. in the 1994 data set [15] ). Instead a new state $\frac{1}{2}^{+}(1750)$ has arisen whose presence makes the above difference. However still in aggrement with Halse [12], we obtain a diquark structure in $\Delta$.

For $\Lambda_{c}$ and $\Sigma_{c}$ we see that the order of the representations is such that it's geometric structure tends to move towards the case (b) ( Fig. 2.). This indicates that for the one heavy quark baryon, the diquark structure exists ; i.e. two light quarks (qq) can form a diquark in (qq-Q) while restoring the $C_{2 v}$ summetry. This is in agreement with the result of Lichtenberg [9]. In the case of $\Xi_{c c}$ baryons, there too is a diquark structure. So one can say 
that in (QQ-q), the two heavy quarks come together to form a diquark. This view is in agreement with others [6, 7, 8, 10]. Here we see that though the diquark structure exists in both the baryons containing one and two heavy quarks, but the nature of the diquark in these two cases is different due to the $C_{2 v}$ symmetry considerations. We are considering only the low angular momentum states so the effect on the diquark structure due to higher angular momentum is not being looked into here.

The three heavy quark baryon $\Omega_{c c c}$ shows the structure of that of the case(c) ( Fig. 2.). This is quite reasonable as all the three quarks are equivalent. This is a new interesting result as it appears that not much work has been done by others in the three heavy quark case. So the conclusion of our model is that the diquark structure exists in one and two heavy quark baryons but not in the three identical heavy quark baryons.

The authors would like to thank the referee for usefull comments. 


\section{References}

[1] W. Kwong, J.L. Rosner and C. Quigg, Ann.Rev.Nucl.Part.Sci. 37, $325(1987)$

[2] J.G. Koerner and H.W. Siebert, Ann.Rev.Nucl.Part.Sci. 41, 511(1991)

[3] N. Isgur and M.B. Wise, Phys.Rev.Lett. 66 (1991) 1130

[4] B. Grinstein, Ann.Rev.Nucl.Part.Sci. 42, 101(1992)

[5] M. Neubert, Phys.Rep C 245, 259(1994)

[6] M.J. Savage and M.B. Wise, Phys.Lett. B 248, 177(1990)

[7] A.F. Falk, M. Luke, M.J. Savage and M.B. Wise, Phys.Rev. D 49, $555(1994)$.

[8] S. Fleck, B. Silvestre -Brac and J.M. Richard, Phys.Rev. D 38, $1519(1988)$.

[9] D.B.Lichtenberg, J.Phys. G 16 1599(1990).

[10] M. Anselmino, E. Predazzi, S. Ekelin, S. Fredriksson and D.B. Lichtenberg, Rev.Mod.Phys. 65, 1199(1993).

[11] F. Iachello, Phys.Rev.Lett. 62, 2440(1989).

[12] P. Halse, Phys.Lett. B 253 9(1991).

[13] A. Abbas, J.Phys G 18, 89(1992) 
[14] C.S. Kalman and B. Tran, Il.Nuovo.Cim. A 102, 835(1989).

[15] Review Of Particle Properties, Phys.Rev.D 50 (1994)1173. 


\section{TABLE CAPTIONS}

Table I: $S U_{f}(3), S U_{f}(4), S U_{f s}(8)$ and $S U_{s}(2)$ levels for the charmed and uncharmed baryons, classified according to isospin (I), strangeness (S) and charm (C).

Table II: Ordering of $[g] L^{\pi}$ ( the notation explained in the text ) representations in the assignment of states for $N, \Delta, \Lambda_{c}, \Sigma_{c}, \Xi_{c c}$ and $\Omega_{c c c}$. (Ground to excited states are ordered from top to bottom.) 


\section{FIGURE CAPTIONS}

Figure 1: Geometrical arrangement of quarks separated by $r_{1}, r_{2}$ in the general case and three other cases as discussed in the text.

Figure 2: Schematic variation of the levels $[g] K$ with the change in the shape of the baryons. Note that $K=0+$ or $0-$ is explained in the text.( Warning: the symbols $+/-$ are not superscript and are not symbols for parity: see text). The vertical axis is labelled by the energy ( schematically) and the horizontal axis is labelled by the values of $r_{1}$ and $r_{2}$ as explained in the text. The dashed lines labeled by $L^{\pi}$ for the negative parity levels and the solid lines for the positive parity levels. The dotted line for the state [3]0 - is for

the baryon containing at least one heavy quark (i.e. not for $N$ and $\Delta$ ). The arrows indicate the location of different baryons as per our assignment. 
Table I

\begin{tabular}{|c|c|c|c|c|c|c|c|}
\hline Particle & I & S & $\mathrm{C}$ & $\begin{array}{l}S U_{f}(3) \\
\left(p_{1} p_{2}\right)\end{array}$ & $\begin{array}{c}S U_{f}(4) \\
\left(\begin{array}{lll}p_{1} & p_{2} & p_{3}\end{array}\right) \\
\end{array}$ & $\begin{array}{c}S U_{f s}(8) \\
{[g]} \\
\end{array}$ & $\begin{array}{c}S U_{s}(2) \\
\text { Spin }\end{array}$ \\
\hline$N$ & $\frac{1}{2}$ & 0 & 0 & $\left(\begin{array}{ll}1 & 1\end{array}\right)$ & $\left(\begin{array}{lll}1 & 1 & 0\end{array}\right)$ & $\begin{array}{c}{[3]} \\
{\left[\begin{array}{ll}2 & 1\end{array}\right]} \\
{\left[\begin{array}{lll}1 & 1 & 1\end{array}\right]} \\
\end{array}$ & $\begin{array}{c}\frac{1}{2} \\
\frac{1}{2}, \frac{3}{2} \\
\frac{1}{2} \\
\end{array}$ \\
\hline$\Delta$ & $\frac{3}{2}$ & 0 & 0 & $\left(\begin{array}{ll}3 & 0\end{array}\right)$ & $\left(\begin{array}{lll}3 & 0 & 0\end{array}\right)$ & $\begin{array}{c}{[3]} \\
{\left[\begin{array}{ll}2 & 1\end{array}\right]} \\
\end{array}$ & $\begin{array}{l}\frac{3}{2} \\
\frac{1}{2} \\
\end{array}$ \\
\hline$\Lambda_{c}$ & 0 & 0 & 1 & $\begin{array}{l}\left(\begin{array}{ll}0 & 1\end{array}\right) \\
\left(\begin{array}{ll}0 & 1\end{array}\right)\end{array}$ & $\begin{array}{l}\left(\begin{array}{lll}1 & 1 & 0\end{array}\right) \\
\left(\begin{array}{lll}0 & 0 & 1\end{array}\right)\end{array}$ & $\begin{array}{c}{[3]} \\
{\left[\begin{array}{ll}2 & 1\end{array}\right]} \\
{\left[\begin{array}{lll}1 & 1 & 1\end{array}\right]} \\
{\left[\begin{array}{ll}2 & 1\end{array}\right]} \\
{\left[\begin{array}{lll}1 & 1 & 1\end{array}\right]} \\
\end{array}$ & $\begin{array}{c}\frac{1}{2} \\
\frac{1}{2}, \frac{3}{2} \\
\frac{1}{2} \\
\frac{1}{2} \\
\frac{3}{2} \\
\end{array}$ \\
\hline$\Sigma_{c}$ & 1 & 0 & 1 & $\begin{array}{l}\left(\begin{array}{ll}2 & 0\end{array}\right) \\
\left(\begin{array}{ll}2 & 0\end{array}\right)\end{array}$ & $\begin{array}{l}\left(\begin{array}{lll}3 & 0 & 0\end{array}\right) \\
\left(\begin{array}{lll}1 & 1 & 0\end{array}\right)\end{array}$ & $\begin{array}{c}{[3]} \\
{\left[\begin{array}{ll}2 & 1\end{array}\right]} \\
{[3]} \\
{\left[\begin{array}{ll}2 & 1\end{array}\right]} \\
{\left[\begin{array}{lll}1 & 1 & 1\end{array}\right]} \\
\end{array}$ & $\begin{array}{c}\frac{3}{2} \\
\frac{1}{2} \\
\frac{1}{2} \\
\frac{1}{2}, \frac{3}{2} \\
\frac{1}{2} \\
\end{array}$ \\
\hline$\Xi_{c c}$ & $\frac{1}{2}$ & 0 & 2 & $\begin{array}{l}\left(\begin{array}{ll}1 & 0\end{array}\right) \\
\left(\begin{array}{ll}1 & 0\end{array}\right)\end{array}$ & $\begin{array}{l}\left(\begin{array}{lll}3 & 0 & 0\end{array}\right) \\
\left(\begin{array}{lll}1 & 1 & 0\end{array}\right)\end{array}$ & $\begin{array}{c}{[3]} \\
{\left[\begin{array}{cc}2 & 1\end{array}\right]} \\
{[3]} \\
{\left[\begin{array}{ll}2 & 1\end{array}\right]} \\
{\left[\begin{array}{lll}1 & 1 & 1\end{array}\right]} \\
\end{array}$ & $\begin{array}{c}\frac{3}{2} \\
\frac{1}{2} \\
\frac{1}{2} \\
\frac{1}{2}, \frac{3}{2} \\
\frac{1}{2} \\
\end{array}$ \\
\hline$\Omega_{c c c}$ & 0 & 0 & 3 & $\left(\begin{array}{ll}0 & 0\end{array}\right)$ & $\left(\begin{array}{lll}3 & 0 & 0\end{array}\right)$ & $\begin{array}{c}{[3]} \\
{\left[\begin{array}{ll}2 & 1\end{array}\right]}\end{array}$ & $\begin{array}{l}\frac{3}{2} \\
\frac{1}{2}\end{array}$ \\
\hline
\end{tabular}


Table II

\begin{tabular}{|c|c|c|c|c|c|}
\hline$N$ & $\Delta$ & $\Lambda_{c}$ & $\Sigma_{c}$ & $\Xi_{c c}$ & \\
\hline$[3] 0^{+}$ & {$[3] 0^{+}$} & {$[3] 0^{+}$} & {$[3] 0^{+}$} & {$[3] 0^{+}$} & {$[3$} \\
{$[21] 0^{+}$} & {$[3] 2^{+}$} & {$[3] 1^{-}$} & {$[3] 1^{-}$} & {$[3] 1^{-}$} & {$[3$} \\
{$[21] 1^{-}(K=0)$} & {$[21] 1^{-}(K=0)$} & {$[21] 0^{+}$} & {$[21] 0^{+}$} & {$[21] 0^{+}$} & {$[21] 1^{-}$} \\
{$[21] 2^{+}$} & {$[21] 0^{+}$} & {$[21] 1^{-}(K=0)$} & {$[21] 1^{-}(K=0)$} & {$[21] 2^{+}$} & {$[21] 1^{-}$} \\
{$[21] 1^{-}(K=1)$} & {$[21] 1^{-}(K=1)$} & {$[3] 2^{+}$} & {$[3] 2^{+}$} & {$[21] 1^{-}(K=0)$} & \\
& & {$[21] 1^{-}(K=1)$} & {$[21] 2^{+}$} & & \\
& & & {$[21] 1^{-}(K=1)$} & & \\
& & & &
\end{tabular}


This figure "fig1-1.png" is available in "png" format from: http://arxiv.org/ps/hep-ph/9605308v1 
This figure "fig1-2.png" is available in "png" format from: http://arxiv.org/ps/hep-ph/9605308v1 